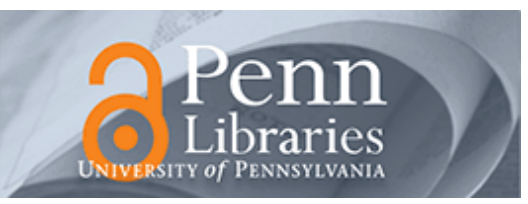

University of Pennsylvania

ScholarlyCommons

March 2003

\title{
Pyrolysis Temperature and Time Dependence of Electrical Conductivity Evolution for Electrostatically Generated Carbon Nanofibers
}

\author{
Yu Wang \\ University of Pennsylvania \\ Jorge J. Santiago-Aviles \\ University of Pennsylvania, santiago@seas.upenn.edu \\ Rogerio Furlan \\ University of Puerto Rico \\ Idalia Ramos \\ University of Puerto Rico \\ Follow this and additional works at: https://repository.upenn.edu/ese_papers
}

\section{Recommended Citation}

Yu Wang, Jorge J. Santiago-Aviles, Rogerio Furlan, and Idalia Ramos, "Pyrolysis Temperature and Time Dependence of Electrical Conductivity Evolution for Electrostatically Generated Carbon Nanofibers", . March 2003.

Copyright 2003 IEEE. Reprinted from IEEE Transactions on Nanotechnology, Volume 2, Issue 1, March 2003, pages 39-43.

Publisher URL: http://ieeexplore.ieee.org/xpl/tocresult.jsp?isNumber=26618\&puNumber=7729

This material is posted here with permission of the IEEE. Such permission of the IEEE does not in any way imply IEEE endorsement of any of the University of Pennsylvania's products or services. Internal or personal use of this material is permitted. However, permission to reprint/republish this material for advertising or promotional purposes or for creating new collective works for resale or redistribution must be obtained from the IEEE by writing to pubs-permissions@ieee.org. By choosing to view this document, you agree to all provisions of the copyright laws protecting it.

This paper is posted at ScholarlyCommons. https://repository.upenn.edu/ese_papers/23

For more information, please contact repository@pobox.upenn.edu. 


\title{
Pyrolysis Temperature and Time Dependence of Electrical Conductivity Evolution for Electrostatically Generated Carbon Nanofibers
}

\author{
Abstract \\ Carbon nanofibers were produced from polyacrylonitrile/N, N-Dimethyl Formamide (PAN/DMF) precursor \\ solution using electrospinning and vacuum pyrolysis at temperatures from $773-1273 \mathrm{~K}$ for $0.5,2$, and $5 \mathrm{~h}$, \\ respectively. Their conductance was determined from $I-V$ curves. The length and cross-section area of \\ the nanofibers were evaluated using optical microscope and scanning probe microscopes, respectively, \\ and were used for their electrical conductivity calculation. It was found that the conductivity increases \\ sharply with the pyrolysis temperature, and increases considerably with pyrolysis time at the lower \\ pyrolysis temperatures of 873,973 , and $1073 \mathrm{~K}$, but varies, less obviously, with pyrolysis time at the higher \\ pyrolysis temperatures of 1173 and $1273 \mathrm{~K}$. This dependence was attributed to the thermally activated \\ transformation of disordered to graphitic carbons.

\section{Keywords} \\ Carbon, conductivity measurement, electrostatic processes, nanotechnology

\section{Comments} \\ Copyright 2003 IEEE. Reprinted from IEEE Transactions on Nanotechnology, Volume 2, Issue 1, March \\ 2003, pages 39-43. \\ Publisher URL: http://ieeexplore.ieee.org/xpl/tocresult.jsp?isNumber=26618\&puNumber=7729 \\ This material is posted here with permission of the IEEE. Such permission of the IEEE does not in any way \\ imply IEEE endorsement of any of the University of Pennsylvania's products or services. Internal or \\ personal use of this material is permitted. However, permission to reprint/republish this material for \\ advertising or promotional purposes or for creating new collective works for resale or redistribution must \\ be obtained from the IEEE by writing to pubs-permissions@ieee.org. By choosing to view this document, \\ you agree to all provisions of the copyright laws protecting it.
}




\title{
Pyrolysis Temperature and Time Dependence of Electrical Conductivity Evolution for Electrostatically Generated Carbon Nanofibers
}

\author{
Yu Wang, Jorge J. Santiago-Aviles, Rogerio Furlan, and Idalia Ramos
}

\begin{abstract}
Carbon nanofibers were produced from polyacrylonitrile/N, N-Dimethyl Formamide (PAN/DMF) precursor solution using electrospinning and vacuum pyrolysis at temperatures from $773-1273 \mathrm{~K}$ for $0.5,2$, and $5 \mathrm{~h}$, respectively. Their conductance was determined from $I-V$ curves. The length and cross-section area of the nanofibers were evaluated using optical microscope and scanning probe microscopes, respectively, and were used for their electrical conductivity calculation. It was found that the conductivity increases sharply with the pyrolysis temperature, and increases considerably with pyrolysis time at the lower pyrolysis temperatures of 873, 973, and $1073 \mathrm{~K}$, but varies, less obviously, with pyrolysis time at the higher pyrolysis temperatures of 1173 and $1273 \mathrm{~K}$. This dependence was attributed to the thermally activated transformation of disordered to graphitic carbons.
\end{abstract}

Index Terms-Carbon, conductivity measurement, electrostatic processes, nanotechnology.

\section{INTRODUCTION}

$\mathbf{E}$ LECTROSTATIC generation, or electrospinning, uses electrostatic forces to spin fibers from a precursor solution. So far, the technique has been mostly used to produce ultrafine polymer fibers [1]-[3]. Chun et al. [4] and Wang et al. [5] pyrolyzed electrospun polyacrylonitrile (PAN) fibers in the vacuum into carbon nanofibers, whose diameter is in the range of $100 \mathrm{~nm}$, much less than that of carbon fibers produced by other methods of spinning [6]-[8]. Wang et al. [9] also synthesized ultrafine piezoelectric (PZT) fibers by sintering electrospun metal alkoxide fibers. Because of their high specific surface area, electrospun ultrafine fibers can be used as high performance filters, scaffolds in tissue engineering, and sensors. These applications become possible only after enough knowledge of the electrostatically generated fibers is gained. However, many properties remain to be investigated. Take electrical conductivity as an example. Although these fibers can be very important in sensor applications, so far only a few investigators have looked into it. Norris et al. [10], using the

Manuscript received August 7, 2002; revised September 24, 2002. This paper was presented in part at the 2nd IEEE Conference on Nanotechnology, August 26-28, 2002, Washington DC. This work was supported by the National Science Foundation Division of Materials Research (NSF-DMR) under Grant 9872689.

Y. Wang and J. J. Santiago-Aviles are with the Department of Electrical and Systems Engineering, University of Pennsylvania, Philadelphia, PA 19104 USA (e-mail: wangyu@seas.upenn.edu).

R. Furlan and I. Ramos are with the Department of Physics and Electronics, University of Puerto Rico, Humacao PR 00791, USA.

Digital Object Identifier 10.1109/TNANO.2003.808510 indirect four-point probe method, measured the conductivity of their electrospun nonwoven ultrafiber mats of polyaniline doped with camphorsulfonic acid blended with polyethylene oxide (PEO). MacDiarmid et al. [11] obtained $I-V$ curves of single $50 \mathrm{wt} \% \mathrm{PAn} . \mathrm{HCSA} / \mathrm{PEO}$ fibers. The lack of conductivity data of electrospun carbon nanofibers can, to a large degree, be attributed to the difficulties in evaluating the cross-section area of the fibers. The authors evaluated the cross-section area using scanning probe microscope (SPM), and measured the room temperature electrical conductivity of PAN-based carbon nanofibers pyrolyzed at $1073 \mathrm{~K}$ [5]. This paper reports pyrolysis temperature and time dependence of the electrical conductivity evolution of carbon nanofibers.

\section{EXPERIMENT}

Commercial PAN powder and N, N-Dimethyl Formamide (DMF) (Aldrich), in a ratio of $800 \mathrm{mg}$ PAN to $10 \mathrm{~cm}^{3} \mathrm{DMF}$, were used to prepare the precursor solution. Thermal gravimetric analysis (TGA) and thermal differential analysis (DTA) were conducted in Argon atmosphere, using a Simultaneous Differential technique module (SDT 29600, TA Instrument), from room temperature to $1573 \mathrm{~K}$ at a heating rate of $10 \mathrm{~K} / \mathrm{min}$.

Single crystal silicon wafers (p-type, $0.1 \Omega \mathrm{cm}$ ), with an silicon oxide layer $\sim 150 \mathrm{~nm}$ thick, were used as substrates. Before the deposition, arrays of $1 \mathrm{~mm} \times 1 \mathrm{~mm}$ or $50 \mu \mathrm{m} \times 50 \mu \mathrm{m}$ gold contacts were fabricated onto the oxidized surface using lithography and vacuum evaporation. The large sheet resistance of silicon oxide makes contacts almost electrically insulated to each other. In fact, there is no detectable conductance between two neighboring contacts without any fiber. Electrospinning was conducted in a homemade setup reported in details previously [5]. The deposition was conducted for a very short time so that only single fibers ran between two neighboring gold contacts.

The as-deposited samples were pyrolyzed in a Brew Model $466-S$ vacuum furnace at $773,873,973,1073,1173$, and $1273 \mathrm{~K}$ for $0.5,2$, and $5 \mathrm{~h}$, respectively. The vacuum was maintained at pressures lower than $1.33 \times 10^{-4} \mathrm{~Pa}$. The substrates, with $50 \mu \mathrm{m} \times 50 \mu \mathrm{m}$ gold contact pad arrays, were used only for fibers pyrolyzed at 773 and $873 \mathrm{~K}$. All other samples, pyrolyzed at higher temperatures, used $1 \mathrm{~mm} \times 1 \mathrm{~mm}$ gold contact pad arrays.

An HP Model 4145B semiconductor parameter analyzer was used to measure the $I-V$ characteristics of the carbon 


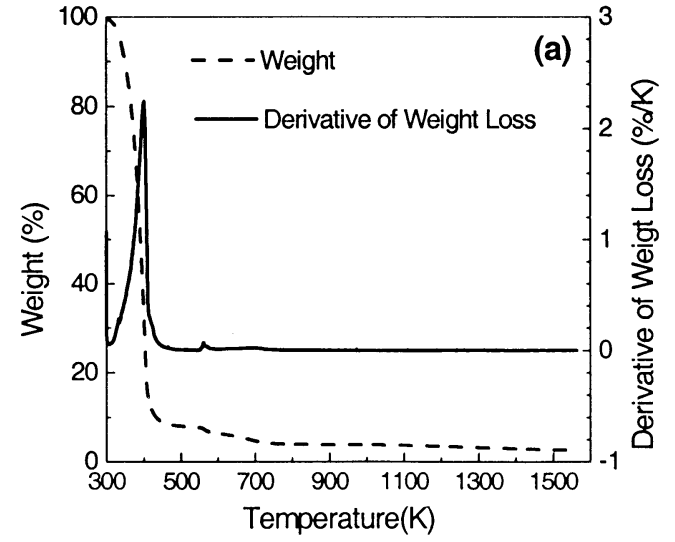

(a)

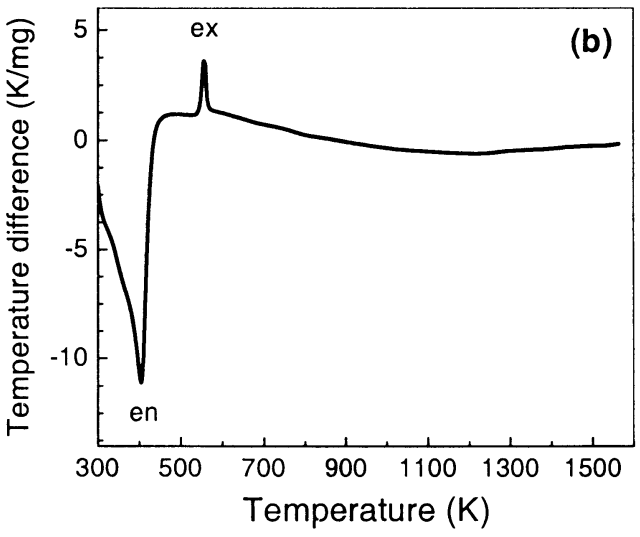

(b)

Fig. 1. (a) TGA and (b) DTA curves of the precursor PAN/DMF solution.

nanofibers at room temperature. The testing voltage ranged from -20 to $20 \mathrm{~V}$, with a step of $0.1 \mathrm{~V}$. Measurements were conducted on the same segment of fiber for 10-20 times under the same conditions.

An optical microscope was used to observe the fiber after pyrolysis and to measure their length $l$. A Digital Instruments Dimension 3000NS-III SPM, operated in tapping mode, was used to record the height and amplitude images of the fibers as data files. Offline image processing software was used to obtain the average cross-section profile, from which cross-section area was evaluated. To ensure reliable results, a scanning electron microscope (SEM) was used to record the image of fibers, from which the horizontal diameter of the imaged fibers was also evaluated. The gold contact pads served as excellent internal length references. The conductivity was finally determined using $\sigma=G l / S$, where $G, S$, and $l$ are conductance, average cross-section area and length of the fibers, respectively.

\section{RESULTS}

The TGA-DTA curves (Fig. 1) show a dramatic weight loss and an endothermic DTA peak between room temperature and $473 \mathrm{~K}$, indicating the solvent (DMF) evaporation. Beyond 473 $\mathrm{K}$, weight decreases at a much slower rate, from $8.34 \mathrm{wt} \%$ at 473 $\mathrm{K}$ to $3.88 \mathrm{wt} \%$ at $873 \mathrm{~K}$, and to $3.35 \mathrm{wt} \%$ and $2.62 \mathrm{wt} \%$ at 1273 and $1573 \mathrm{~K}$, respectively. The exothermic DTA peak centered

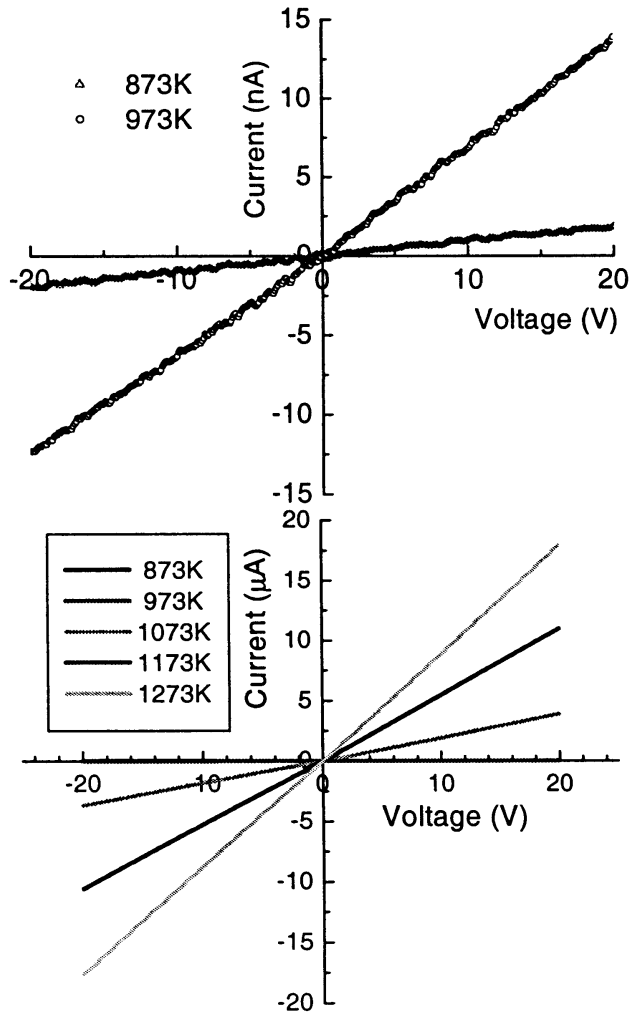

Fig. 2. $I-V$ curves of carbon nanofibers pyrolyzed at different temperatures.

around $570 \mathrm{~K}$ [Fig. 1(b)] is produced by PAN decomposition [12].

Fig. 2 shows the $I-V$ curves of carbon fibers pyrolyzed at different temperatures for $0.5 \mathrm{~h}$. Their linearity implies an ohmic contact between the contact pad and the fibers. The linear slope, or the conductance of the fibers $G$ increases sharply with the pyrolysis temperature.

Fig. 3 shows SPM height and amplitude images of a typical single fiber. The apparent semicircular profile of the cross-section [Fig. 3(c)] may be misleading because the cone-shaped tip could not track the lower half of the cross-section [Fig. 3(d)]. However, the height of the fiber, or the vertical diameter, is real. The full width at half maximum (FWHM) was taken as the horizontal diameter of the fibers, since the measured values of vertical and horizontal diameters on the same cross-section are different, and the cross-section is elliptical rather than circular, as previously supposed.

The SEM image of the fiber revealed by SPM in Fig. 3 clearly shows its irregularity (Fig. 4). The average horizontal diameter of the fiber was measured to be $120 \mathrm{~nm}$, approximately the same as the FWHM value obtained from SPM cross-section profile, justifying the use of FWHM as the horizontal diameter. This paper uses the diameter values measured from SPM height images since they give both horizontal and vertical diameters.

Pyrolysis at $773 \mathrm{~K}$ did not generate a conductance high enough as to be detected. Since the detection limit of the measurement system is estimated to be $0.2 \mathrm{~S} / \mathrm{m}$, the conductance of the fibers pyrolyzed at $773 \mathrm{~K}$ must be lower than this value. At temperatures higher than $873 \mathrm{~K}$, the conductivity 

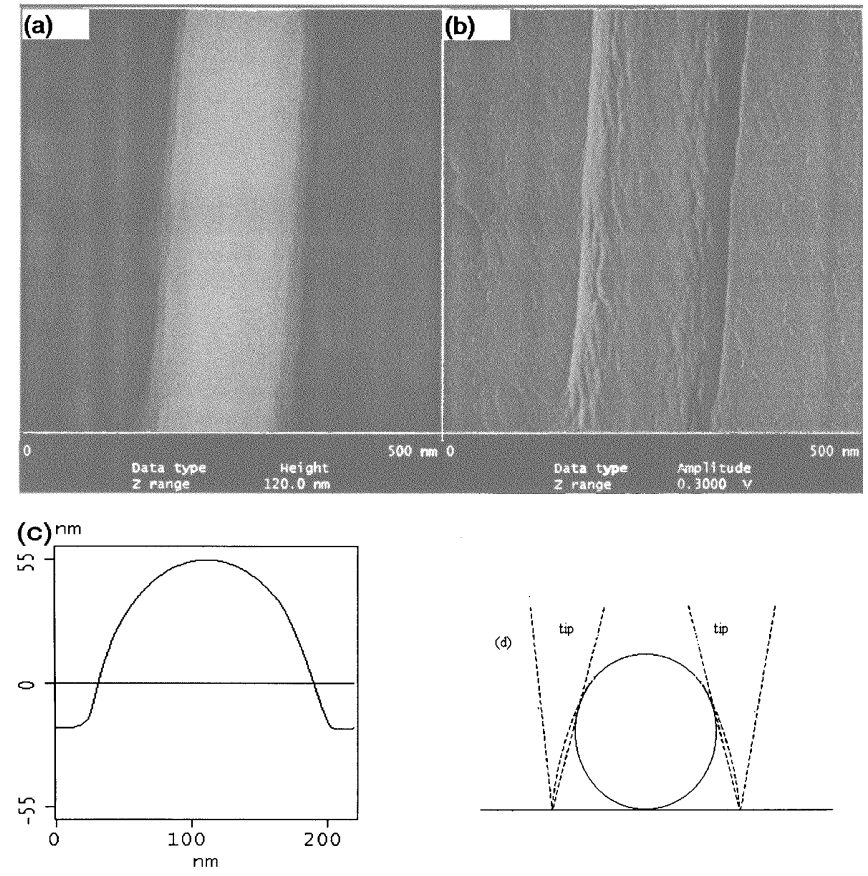

Fig. 3. SPM micrograph of carbon nanofiber and its cross-section profile.

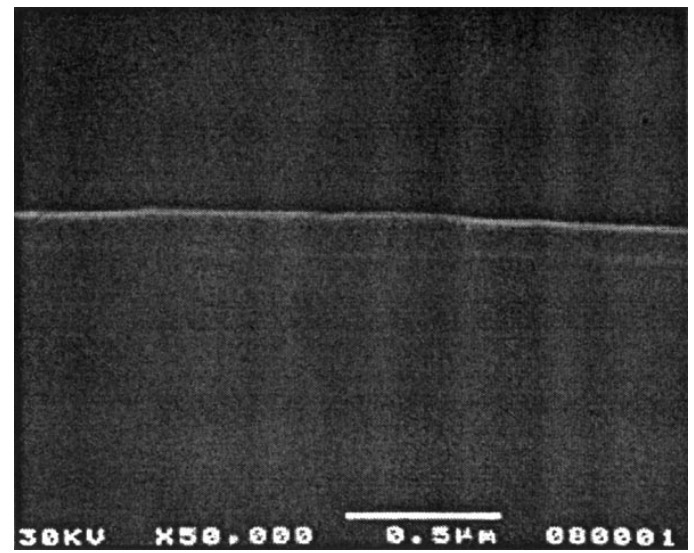

Fig. 4. SEM image of the same fiber revealed by SPM in Fig. 3.

increases sharply with pyrolysis temperature. It also increases with the pyrolysis time at lower pyrolysis temperatures $(873$, 973, and $1073 \mathrm{~K})$ [Fig. 5(a)], but levels off at higher pyrolysis temperatures (1173-1273 K) [Fig. 5(b)]. In fact, at the pyrolysis temperature of $1173 \mathrm{~K}$, the conductivity decreases slightly with the pyrolysis time. Such abnormal independence may be attributed to the increased porosity, and needs further investigation.

\section{DISCUSSIONS}

\section{A. Error Analysis}

The error in conductivity measurement arose mainly from two sources: 1) the contact resistance between the fibers and the contact pads, and 2) the irregularities of the fibers, and consequently, the difficulties in the evaluation of the cross-section area. To reduce the effect of the contact resistance, gold contact pads were used. They were heat-treated together with the fibers during the vacuum pyrolysis. The diffusion of carbon and gold atoms between the contacts and the fibers not only enabled an ohmic contact (Fig. 2), but also reduced the contact resistance. Furthermore, the distance between the neighboring contacts was intentionally kept not too small: $50 \mu \mathrm{m}$ for fibers pyrolyzed at 773 and $873 \mathrm{~K}$ and $1 \mathrm{~mm}$ for fibers pyrolyzed at higher temperatures, so that the resistance of the fibers itself is much larger than the contact resistance. Fiber samples too irregular were discarded for reliable measurement, and the average cross-section profiles were used to evaluate the cross-section area.

\section{B. Change During the Pyrolysis of the Precursor Fiber}

The pyrolysis process in this paper differs from the general case in two respects: the precursor solution contains 92\% (weight) DMF and the precursor fibers contain some residual DMF even if the solvent mostly evaporated during the electrospinning; the precursor fibers were pyrolyzed in the vacuum, where the residual oxygen content is minimized. The dramatic weight loss and endothermic process between room temperature and $473 \mathrm{~K}$, shown by TGA and DTA curves (Fig. 1), indicates the evaporation of DMF. Above $473 \mathrm{~K}$, the weight remains about $8.34 \mathrm{wt} \%$, very close to the PAN concentration of the precursor solution. Considering the fact that the volatile DMF kept evaporating as soon as it is loaded into the pan, and before starting the analysis, it is reasonable to think that DMF has totally evaporated by $473 \mathrm{~K}$. Although the DMF content of our precursor solution and precursor fibers are quite different, the TGA-DTA curves of the precursor solution, shown in Fig. 1, may truly represent the pyrolysis change of precursor fibers at temperatures higher than $473 \mathrm{~K}$.

During the complicated pyrolysis process, PAN generally goes through stabilization between 473 and $673 \mathrm{~K}$, and carbonization at higher temperatures. Stabilization involves dehydrogenation, cyclization and, if in the air, oxidation. The exothermic process is due to the uncontrolled thermal polymerization of the nitrile group with the release of the heat of polymerization. During carbonization the fibers lose noncarbon elements, as well as partial carbon, in the form of volatile byproduct gases, such as $\mathrm{HCN}, \mathrm{NH}_{3}$, and $\mathrm{H}_{2}$, and a graphite-like structure is formed. The basic structural unit (BSU) of carbon fibers consists of a stack of conductive turbostratic layers. The BSU can split, twist, fold, and join other BSUs to form microdomains, which can also split, twist, fold, and join, etc., to form carbon fibers. Thus, the fine structure of carbon fibers is not a homogeneous, monolithic carbon but rather a somewhat chaotic collection of BSUs formed into microdomains interspersed with uncarbonized intermediate products and pores [6]-[8]. At low temperatures, or immediately after stabilization, the oriented BSUs are isolated, and the fibers are not conductive. After the disappearance of the heteroatoms, which is believed to take place between 600 and $873 \mathrm{~K}$, isolated columns of materials are formed, followed by microdomains as the columns form more coherent structures. Once the microdomains become continuous, and the layers of turbostratic carbon appear across the fibers, constituting a 


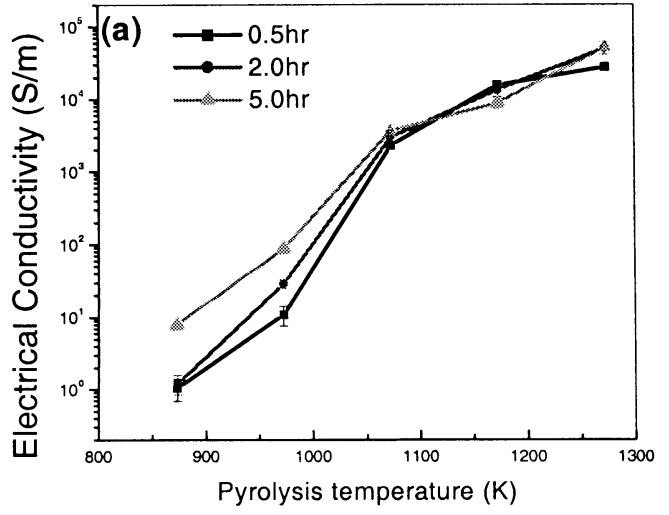

(a)

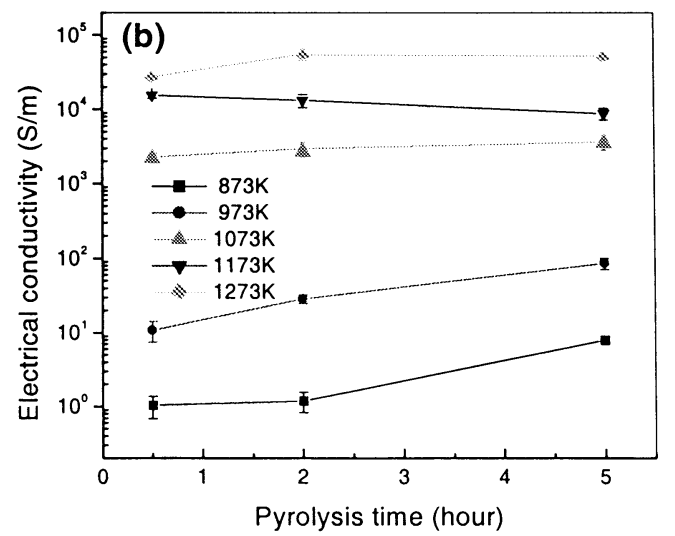

(b)

Fig. 5. Pyrolysis (a) temperature and (b) time dependence of the conductivity.

conducting channel, the fibers start to be conducting. This takes place around $873 \mathrm{~K}$.

The formation of graphite carbon at near $873 \mathrm{~K}$ was confirmed by an earlier Raman scattering investigation. Raman spectra showed coexistence of graphitic and disordered carbons [5]. It is interesting that although weight decreases only slightly from $3.88 \%$ at $873 \mathrm{~K}$ to $3.35 \%$ at $1273 \mathrm{~K}$, the conductivity increases by almost five orders of magnitude. It is strongly suggested that the change in this temperature range is not mainly the extraction of heteroatoms but rather the transformation of disordered carbons and isolated BSUs to continuous graphite domains, or the early stage of graphitization. This process is a kinetically thermally activated process. The higher pyrolysis temperature and/or the longer pyrolysis time, the higher graphite molar fraction in the pyrolyzed carbon fibers. So, the increase of pyrolysis temperature results in a sharp increase in the conductivity [Fig. 5(a)]. The effect of time is more pronounced at lower temperatures than at higher temperatures, because it takes a longer time for the system to reach its quasiequilibrium state at lower pyrolyzing temperatures than at higher temperatures.

\section{CONCLUSION}

The conductivity of PAN-based carbon nanofibers produced by electrospinning was measured. It increases sharply with the pyrolysis temperature, and also increases considerably with pyrolysis time at lower pyrolysis temperatures $(873,973$, and 1073 $\mathrm{K})$, but varies less obviously with pyrolysis time at higher pyrolysis temperatures (1173 and $1273 \mathrm{~K}$ ).

\section{REFERENCES}

[1] A. Formhals, "Process and apparatus for preparing artificial threads," U.S. Patent 1975 504, Oct. 2, 1934.

[2] J. Doshi and D. H. Reneker, "Electrospinning process and application of electrospun fibers," J. Electrost., vol. 35, pp. 151-156, Aug. 1995.

[3] D. H. Renker and I. Chun, "Nanometer diameter fibers of polymer, produced by electrospinning," Nanotechnol., vol. 7, pp. 216-223, Sept. 1996

[4] I. Chun, D. H. Reneker, H. Fong, X. Famg, J. Deitzel, N. B. Tan, and $\mathrm{K}$. Kearns, "Carbon nanofibers from polyacrylonitrile and mesophase pitch," J. Adv. Mater., vol. 31, pp. 36-41, 1996.

[5] Y. Wang, S. Serrano, and J. J. Santiago-Aviles, "Conductivity measurement of electrospun PAN-based carbon nanofiber," J. Mater. Sci. Lett., vol. 21, pp. 1055-1057, July 2002.

[6] L. H. Peebles, Carbon Fibers. Boca Raton, FL: CRC, 1995, pp. 7-26.

[7] O. P. Bahl, Z. Shen, J. G. Lavin, and R. A. Ross, "Manufacture of carbon fibers," in Carbon Fibers, 3rd ed, J.-B. Donnet et al., Eds. New York: Marcel Dekker, 1998, ch. 1, revised and expanded, pp. 1-84.

[8] J.-P. Issi and B. Nysten, "Chapter 6 electrical thermal transport in carbon fibers," Carbon Fibers, pp. 371-462.

[9] Y. Wang, S. Serrano, and J. J. Santiago-Aviles, "Electrostatic synthesis and characterization of $\mathrm{Pb}\left(\mathrm{Zr}_{x} \mathrm{Ti}_{1-x}\right) \mathrm{O}_{3}$ micro/nano-fibers," in Proc. MRS Fall Meet.—Symp. U: Advanced Fibers, Plastics, Laminates and Composites, Boston, MA, Nov. 25-31, 2001, pp. 359-364.

[10] I. D. Norris, M. M. Shaker, F. K. Ko, and A. G. MacDiarmid, "Electrostatic fabrication of ultrafine conducting fibers: Polyaniline/polyethylene oxide blends," Synthetic Metals, vol. 114, pp. 109-114, Aug. 2000.

[11] A. G. MacDiarmid et al., "Electrostatically-generated nanofibers of electronic polymers," Synthetic Metals, vol. 119, pp. 27-30, Mar. 2001.

[12] E. V. Thompson, "The thermal behavior of acrylonitrile polymers I. On the decomposition of polyacrylonitrile between 250 and $325^{\circ} \mathrm{C}$," Polymer Lett., vol. 4, pp. 361-366, 1966.

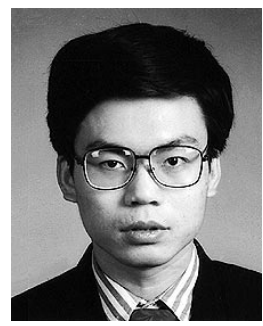

Yu Wang received the B.S. degree in materials science and engineering from Shanghai Jiaotong University, Shanghai, China, in 1988 and the M.S. degree in electrical engineering from the University of Pennsylvania, Philadelphia, in 2001, where he is currently working toward the Ph.D. degree in the Department of Electrical and Systems Engineering.

His current research interest is micro- and nanofibers.

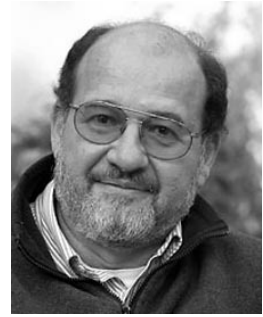

Jorge J. Santiago-Aviles is currently an Associate Professor of electrical and system engineering at the University of Pennsylvania, Philadelphia. He has done research work in II-VI semiconducting compounds for LEDs, carbon and graphite composites, silicon sensors and actuators, electroceramic devices and LTCC tape based sensing and actuating devices.

His research interest spans the interface between electronic devices and materials science. Currently, his interests are focused on the field of nanostructures and nanomaterials. 


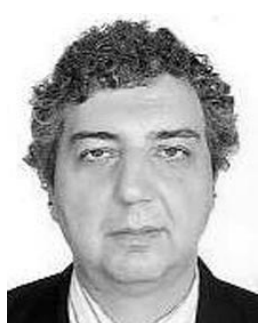

Rogerio Furlan received the electrical engineering degree from the Faculdade de Engenharia Industrial (FEI), Brazil, in 1982, the M.S. and Ph.D. degrees in electrical engineering from the University of Sao Paulo, Sao Paulo, Brazil, in 1985 and 1990, respectively.

From 1988 to 2001, he was a Faculty Member of the Polytechnic School of the University of Sao Paulo, becoming an Associate Professor in 1998. Since 1981, he has been a member of the Laboratory of Integrated Systems of the Polytechnic School of University of Sao Paulo, where, from 1989 to 1984, he coordinated the Metallization Group and, from 1996 to 2001, the Microstructures Group. In August 2001, he became an Associated Professor of the Physics and Electronics Department of the University of Puerto Rico at Humacao. His primary areas of interest are micro- and nanofibers and microfluidic devices.

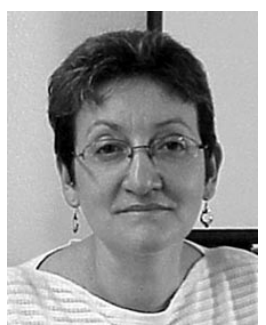

Idalia Ramos received the B.S. degree in physics from the University of Puerto Rico at Humacao, in 1983 and the M.S. degree in electrical engineering from the University of Pennsylvania, Philadelphia, in 1986.

Currently, she is an Associate Professor in the Department of Physics and Electronics at the University of Puerto Rico, Humacao. Her research is in the area of materials and implementation of micro-electro-mechanical devices, micro- and nanofibers. She is also the Program Director of a Collaborative to Integrate Research and Education between the University of Puerto Rico and the University of Pennsylvania and the Program Director of ADVANCE Institutional Transformation, a program to change the status of women in the science faculty of the University of Puerto Rico at Humacao. 